Bull. Mater. Sci., Vol. 4, No. 2, April 1982, pp. 125-132. (C) Printed in India.

\title{
Motion induced by surface-tension gradients
}

\author{
SIMON OSTRACH \\ Case Western Reserve University, Cleveland, Ohio 44106, USA \\ MS received 19 June 1981
}

\begin{abstract}
The physical mechanisms of flows generated by surface-tension gradients are clearly defined and the relevant dimensionless parameters are derived. These are used to indicate the qualitative nature of possible flows.
\end{abstract}

Keywords. Surface-tension gradient; dimensionless parameters ; Marangoni instability ; thermocapillarity; diffusocapillarity.

\section{Intraduction}

It has been indicated in the carlier article by the author in these procecdings that the surface-tension gradients can induce fluid flow in a reduced grevity environment (Ostrach 1977) or modify the existing flow. Depending upon whether the gradient is caused by gradients in temperature, composition or electric potential, the ensuing flow is referred to as thermocapillary, diffusocapillary or thermoelectric flow, respectively (Scriven 1974). The flow could be of two types (Scriven 1974) as is also the case with buoyancy driven convention. If one of the above gradients is perpendicular to the interface, a Marangoni instability can occur, under proper conditions, leading to cellular flows, analogous to unstable convection induced by buoyancy under normal gravity. Although the Marengoni instability is also referred to (Sternling and Scriven 1959 ; Kenning 1968) as a form of interface turbulence, the flow obtained is laminar. This flow, however, can become turbulent under proper conditions (Schmidt and Milverton 1935).

Since temperature or concentration gradients can cause gradients in surface tension as well as density, buoyancy and surface-tension driven flows can occur simultaneously. However, the relative importance of the two mechanisms is governed by the value of gravitational accelcration and the size of the fluid body as is evident from the Bond number

$$
B_{0}=\rho g d^{2} / \sigma,
$$

where $\rho$ is density, $g$ the acceleration due to gravity, $d$ a characteristic linear dimension and $\sigma$ the surface tension. The surface tension becomes important in 
deciding the shape and stability of surfaces or interfaces and fluid flows in liquids of larger configuration only in reduced gravity environment. Therefore, most existing work on the effect of surface tension deals with motions in liquids of smallar configuration, such as, with flows in capillary tubes or thin films or the mation of droplets or bubbles or short wavelength water waves. Within the limits imposed by this restriction there are, nevertheless, many technologically important processes in which surface tension can be significant. The excellent summaries by Kenning (1968) and Levich and Krylov (1969) outline the many types of problems treated covering such applications as boiling heat transfer, spreading of films such as oil and paint, wave phenomena, jet decay, and corrosion problems. Surface tension was also studied as a mechanism of flame spreading (Sirignano and Glassman 1970).

The effect of surface tension on large sized fluid bodies under reduced ar microgravity environment is receiving researchers' focussed attention only now. Perhaps the most unique aspect of the reduced gravity environment in a spacecraft is that it offers this possibility of containerless processing of materials so that contamination or defects due to container reactions or interactions can be eliminated. There are other advantages to the containerless handling of liquids, such as, longer stable lengths of floating liquid zones. Liquids and molten metals with free surfaces are inherent to all containerless processes, and their nature must be well understood to take full advantage of that novel processing scheme. In particular, the shape of the bulk fluid under various conditions and its stability to changes must be predictable. Furthermare, from equation (1) it appears that surface tension is important under micro-gravity conditions with essentially no limitations on the configuration scale. Thus, the details of the surface-tension induced flows and the transpart processes within the fluids over ranges of conditions must also be known. In addition, surface and bulk constitutive properties must be known with accuracy. Unfortunately, there exists little information of this kind for configurations and conditions that would be applicable to space processing.

\section{Dimensionless parameters}

Estimates of the relation between the two flow mechatisms have been previously obtained from the Bond number, equation (1). However, the limits of applicability of the Bond number is unzertain so that a more general criterion is required. Such a criterion (dimensionless parameter) would also be extremely valuable to see which, if any, space-related phenomena could be simulated on earth. It is essential to determine the relevant dimensionless parameters that describe complex phenomena, because they indicate the dominant physical factors, mathematical simplifications, data correlations, and proper theoretical and experimental models. Furthermore, the dimengionless parameters permit order of magnitude estimates to be made so that the qualitative features of the phenomena can be determined. The parameters can be obtained in several ways, but, to obtain all of the information from them, it is best to derive them from the basic equations and boundary conditions that describe the phenomena. This is done by making all variables not only dimensionless, but also of unit order of magnitude (Ostrach 


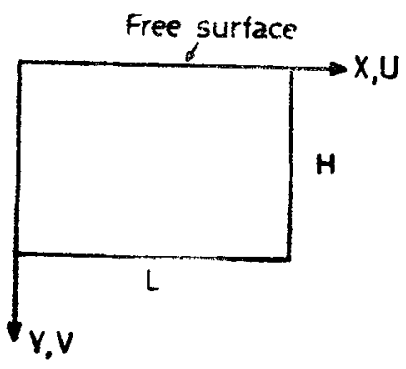

Figure 1. Configuration.

1966). The normalization of the variables is not an automatic process, but requires sufficient physical information or jnsight to choose the prcper reference quantities. It was surprising to find from an extensive, but parhaps not complete, review of the literature on surface-tension induced flows, that although some authors, for example, Levich (1962), Kenning (1968) and Stanck and Szekely (1964) indicate, or imply, a physically reasonable rcference velocity, there daes not appear to be an explicit derivation of the parameters bascd on such a reference. Furthermore no classification of problems or analyses based on the parameters could be found. Therefore, the derivation will be outlined herein and the results will be comparcd to existing ones, and the implications for future work will be indicated.

For convenience only, consideration will be given to a two-dimensional rectangular container which is filled with a quasi-incompressible liquid with the upper surface free (see figure 1). Quasi-incompressible means that the liquid density is taken to be constant except in the body force term which can then be written as a buayancy force. The surface-tension variation is considered to be induced by a temperature variation, recognizing that the development for diffusocapillarity is similar. Therefore, the flow is assumed to be steady and will be described by the basic equations that express the conscrvation of mass, momentum, and energy. To normalize the variables let

$$
\begin{aligned}
& x=X|L, y=Y| H, u=U\left|U_{R}, v=V\right| U_{R}(H / L), \\
& p=\frac{P}{\rho U_{R}^{2}}, \quad \tau=\frac{T-T_{c}}{T_{v}-T_{c}},
\end{aligned}
$$

wherc capital letters denote dimensional quantitics and the lowcr casc letters dimensionless oncs ; also $X$ and $Y$ are the coordinates indicated in figure $1, L$ is the container length, $H$ is the liquid depth, $T_{w}$ and $T_{c}$ are the hot and cold wall temperatures, respectively, $P$ is the fluid pressure, $\rho$ is the density, and $U$ and $V$ are the velosity components. Note that with the exception of the velocity components (and, possibly the pressure) all the variables as expressed in (2) are clearly not only dimensionless but also of unit order of magnitu de i.e., they are normalized as well. The dimensionless basic equations obteined by us of equation (2) are

$$
\frac{\partial u}{\partial x}+\frac{\partial v}{\partial y}=0
$$




$$
\begin{aligned}
& u \frac{\partial u}{\partial x}+v \frac{\partial u}{\partial y}=\frac{1}{\operatorname{Re} A^{2}}\left(A^{2} \frac{\partial^{2} u}{\partial x^{2}}+\frac{\partial^{2} u}{\partial y^{2}}\right)-\frac{\partial p}{\partial x} \\
& u \frac{\partial v}{\partial x}+v \frac{\partial v}{\partial y}=\frac{1}{\operatorname{Re} A^{2}}\left(A^{2} \frac{\partial^{2} v}{\partial x^{2}}+\frac{\partial^{2} v}{\partial y^{2}}\right)-\frac{\mathrm{Gr}}{\operatorname{Re}^{2} A} \tau-\frac{1}{A^{2}} \frac{\partial p}{\partial y} \\
& u \frac{\partial \tau}{\partial x}+v \frac{\partial \tau}{\partial y}=\frac{1}{\operatorname{Pr} \operatorname{Re} A^{2}}\left(A^{2} \frac{\partial^{2} \tau}{\partial x^{2}}+\frac{\partial^{2} \tau}{\partial y^{2}}\right)
\end{aligned}
$$

where the Reynolds number is $\operatorname{Re}=U_{R} L / v$, the Grashof number is $\mathrm{Gr}=\beta g\left(T_{w}-T_{c}\right) L^{3} / v^{2}$, the aspect ratio is $A=H / L$, and the Prandtl number is $\operatorname{Pr}=v / \alpha$ with $\beta$ the fluid volumetric expansion coefficient, $g$ the acceleration due to gravity, $v$ the kinematic viscosity, and $a$ the thermal diffusivity.

An appropriate reference, $U_{R}$, must now be determined for the velocity in order to normalize it. In every existing analysis of surface-tension flows it appears that either the ratio $v / L$ or $\alpha / L$ has been used as the reference velocity. The first one implies that inertia and viscous forces are of the same order of magnitude and the second that conduction and convection are of the same order. Neither of these is necessarily true in many problems of intercst. These ratios do, indecd, have the dimensions of velocity but they do not normalize the velocity. The driving mechanism for the flow is the shear stress induced at the free surfacc by the surfacetension gradient. Thurefore, for surface-tension flows the scale of the velocity must be obtained from the balance of the tangential stresses at the frec surface. Although this was suggested by Kenning (1968) it was not properly applicd nor ultimately used.

The tangential stress balance at the free surface can be written as

$$
-\mu \frac{\partial U}{\partial Y}=\frac{\partial \sigma}{\partial X}=\frac{\partial \sigma}{\partial T} \frac{\partial T}{\partial X}
$$

where $\mu$ is the absolute viscosity and $\sigma$ the surface tension. If $\operatorname{Re} A^{2} \leqslant 1$ it can be seen from (4) and (5) that inertia effects will be negligible and the flow will be a viscous type. Therefore, the effects of surface tension will penetrate downward into the fluid by viscosity and $h$ is the proper length scale for $Y$. Thus, substitution of (2) into (7) yields

$$
-\frac{\partial u}{\partial y}=\frac{(\partial \sigma / \partial T)\left(T_{w}-T_{c}\right)}{\mu U_{R} L} H \frac{\partial \tau}{\partial x}
$$

which, for both terms to be of the same order,

$$
U_{R}=\frac{(\partial \sigma / \partial T)\left(T_{10}-T_{c}\right)}{\mu} \frac{H}{L}
$$

Equation (8) together with the inequality for this case indicates the configuration conditions for such flows to occur viz.,

$$
\stackrel{h}{L} \ll\left(\frac{\mu v}{(\partial a / \partial T)\left(T_{w}-T_{0}\right) H}\right)^{1 / 2} \equiv \frac{1}{\sqrt{R_{\sigma}}} .
$$

The surface-tansion Reynolds number, $R_{\sigma}$, defined here is similar to that given in Kenning (1968) and Stanck and Szekely (1964). Levich (1962) presented equivalent expressions to (8) and (9). 
On the other hand, if $\operatorname{Re} A^{2} \gg 1$ a boundary layer flow will occur and the viscous and inertia terms must be of the same order therein. The boundarylayer thickness, $\delta$, is the appropriate length scale for this case and it can be found by a coordinate stretching to be

$$
\delta / H=1 / A(\operatorname{Re})^{1 / 2} .
$$

From the free-surface tangential stress balance and (10) it follows that

$$
U_{R}=\frac{(\partial \sigma / \partial T)\left(T_{10}-T_{c}\right)}{\mu} \frac{\delta}{L}=\frac{(\partial \sigma / \partial T)\left(T_{w}-T_{c}\right)}{\mu}\left(v / U_{R} L\right)^{1 / 2}
$$

so that

$$
U_{R}=\left(\frac{(\partial \sigma / \partial T)^{2}\left(T_{10}-T_{e}\right)^{2} v}{\mu^{2} L}\right)^{1 / 3}
$$

Note that both reference velocities derived, (8) and (11), are expressed in terms of the variables that are the physically important ones for establishing such flows.

For boundary layer flows it can be found that

$$
H / L \gg 1 /\left(R_{\sigma}\right)^{1 / 2} \text {. }
$$

With the reference velocities determined, the dimensionless equations are : For the viscous case

$$
\begin{aligned}
& R_{\sigma} A^{2} \ll 1\left[H / L \ll 1 /\left(R_{\sigma}\right)^{1 / 2}\right] \\
& 0=A^{2} \frac{\partial^{2} u}{\partial x^{2}}+\frac{\partial^{2} u}{\partial y^{2}}-\frac{\partial p}{\partial y}, \\
& 0=A^{2} \frac{\partial^{2} v}{\partial x^{2}}+\frac{\partial^{2} v}{\partial y^{2}}-\frac{\mathrm{Gr} A}{R_{\sigma}} \tau-\frac{1}{A^{2}} \frac{\partial p}{\partial y}, \\
& \text { Ma } A^{2}\left(u \frac{\partial \tau}{\partial x}+v \frac{\partial \tau}{\partial y}\right)=A^{2} \frac{\partial^{2} \tau}{\partial x^{2}}+\frac{\partial^{2} \tau}{\partial y^{2}},
\end{aligned}
$$

where for this case the appropriate reference pressure is $\mu U_{R} L / H^{2}$ rather than $\rho U_{R}{ }^{2}$. The left side of (6?) can also be neglected unless

$$
\operatorname{Pr} \geqslant 1 / R_{\sigma} A^{2} \text {. }
$$

For the boundary-lajcr case $R_{\sigma} A^{2} \gg 1\left(H L \gg 1 / R_{\sigma}\right)$

$$
\begin{aligned}
& u \frac{\partial u}{\partial x}+v \frac{\partial u}{\partial y}=\left(A / R_{\sigma}\right)^{2 / 3} \frac{\partial^{2} u}{\partial x^{2}}+\frac{\partial^{2} u}{\partial y^{2}}-\frac{\partial p}{\partial x}, \\
& u \frac{\partial v}{\partial x}+v \frac{\partial v}{\partial y}=\left(A / R_{\sigma}\right)^{2 / 3} \frac{\partial^{2} v}{\partial x^{2}}+\frac{\partial^{2} v}{\partial y^{2}}-\frac{\operatorname{Gr} A}{R_{\sigma}} \tau-\left(R_{\sigma} / A\right)^{2 / 3} \frac{\partial p}{\partial y}, \\
& u \frac{\partial \tau}{\partial x}+v \frac{\partial \tau}{\partial y}=\frac{1}{\operatorname{Pr}}\left[\left(A / R_{\sigma}\right)^{2 / 3} \frac{\partial^{2} \tau}{\partial x^{2}}+\frac{\partial^{2} \tau}{\partial y^{2}}\right]
\end{aligned}
$$

where the Marangoni number, $\mathrm{Ma}=\operatorname{Pr} R_{\sigma}$, is a modified Pclet number. Equivalent expressions follow for diffusocapillary flows. For Prandtl numbers different from unity, the velocity and temperature boundary layers will be unequal.

The situation derived above is analogous to that in natural convection. In the latter, different reference velocitics are required for different force balances (Ostrach 1964) and the resulting equations contain the parameters to various powers. 
The influence of buoyancy for each case is determined from the coefficient of the buoyancy term in (13). Explicitly, for viscous flows

$$
\text { Gr } A / R_{\sigma}=\rho \beta g L^{2} /(\partial \sigma / \partial T) \equiv \bar{B}_{0},
$$

where $\bar{B}_{0}$ is a modificd Bond number. For boundary-layer flows, however, the buoyancy term must be compared to the highest order term in its equation (the pressure gradient) so that

$$
\text { Gr } A^{5 / 3} / R_{\sigma}^{5 / 3}=\bar{B}_{0}\left(A / R_{\sigma}\right)^{2 / 3} \text {. }
$$

Note that buoyancy is negligible in a sufficiently reduced gravity enviranment. The various options for reducing buoyancy an earth are indicatcd in (19) and (20).

With the normalization presented above no dimensionless parameters appear in the boundary conditions. The dimensionless cquations utilised in the existing analyses of surface-tension flows that are based on $U_{R}=v / L$ have only $\mathrm{Gr}$ as a factor of the buoyancy term and $\left(1 / \mathbf{P}_{\mathrm{r}}\right)$ as a factor of the conduction term. The situation for diffusocapillarity with $U_{R}=\alpha / L$ is similar, with the $\mathrm{Sc}$ hmidt number, $v / D$ replacing the Prandtl number in the factor of the diffusion term. No other parameters appear in the basic equations. However, $R_{\sigma}$ appears as a factor of the surface-tension gradient in the free-surface tangential strcss boundary condition. For extreme values of $R_{\sigma}$ the proper boundary condition could be lost. In that formulation the Marangoni nimber docs nat appear explicitly. From (4) to (6) it should be evident that flow quantitios are related to $R_{\sigma}$ and transported to Ma.

If no terms in the equations are to be neglectcd (as in numerical solutions) any non-dimensionalization can be used although thre are drfinite a.dvantages to working with unit-order variables that are obtained by normalization. However, to obtain a qualitative view. of the phenomena or to simplify the equations by ordering procedures it is essential that the equations be normalized. If this is not done either terms will be incorrectly neglected or retained; the latter usually unduly extends computing time at the least. With proper normalization the order of magnitude of each term is indicated by its coefficient (dimcnsionless parameter) and comparison among terms is possible. Such a procedure enables one to know explicitly the conditions under which the simplifications are valid. With equations (4) to (6) in the form presented herein (which is the same as for usual fluid problems) the qualitative nature of the flow and transport can be determined before the equations are solved by evaluating the dimrnsionless par?metcrs for the specific cases of intorest. Note that thermocapillary flow problc ms with buayancy are defined by four parameters, viz., $R_{\sigma}, \mathrm{Ma}$, $\mathrm{Gr}$, and $A$. It is interesting to note from the definitions of (9), (19) and (20), that aside from length scalcs and the imposed temperature differerce the parameters arc call given in terms of thermophysical properties. Estimates of the parameters are presented in table 1 for length scales of $10 \mathrm{~cm}$, temperature differences of $50^{\circ} \mathrm{C}$, and an aspect ratio of unity.

If $R_{\sigma}<1$ the flow will be of a creeping or highly viscous type and inertial effects will be negligible. Such flows appear to be possible only with very viscous fluids like oils and glass. If $\mathrm{Ma}<1$ also, the heat transfer will be solely due to conduction. For $R_{\sigma}>1$ flow boundary layers can be expected. If Ma $>1$ there will alsa be a temperature boundary layer (of a different extent if $\operatorname{Pr} \neq 1$ ). These considerations (together with the ones concerning the configuration geometry) can 
Table 1. Parametric values for length scales of $10 \mathrm{~cm}$, temperature differences of $50^{\circ} \mathrm{C}$ and an aspect ratio of unity.

\begin{tabular}{llllll}
\hline & $R_{\sigma}$ & $\mathrm{Gr}$ & $\mathrm{Ma}$ & $\bar{B}_{\sigma}=\mathrm{Gr} / R_{\sigma}$ & $\bar{B}_{0} / R_{\alpha}{ }^{2 / 3}$ \\
\hline Silicone oils & $10^{-1}-10^{4}$ & $10^{2}-10^{7}$ & $10^{3}-10^{6}$ & $10^{3}$ & 10 \\
Glass & $10^{-1}$ & 10 & $10^{2}$ & $10^{2}$ & $\ldots$ \\
Water & $10^{6}$ & $10^{8}$ & $10^{7}$ & $\ldots$ & $10^{-2}$ \\
Liquid metals & $10^{5}-10^{6}$ & $10^{8}-10^{10}$ & $10^{3}-10^{5}$ & $\ldots$ & $10^{-2}$ \\
\hline
\end{tabular}

either lead to sufficient mathomatical simplifications so that analytical solutions can be obtained or else they cain indicate regions in which finer grids are required for numerical solutions. From the table it can be seen that a large range of problems is possible. Furthermore, bu oyancy is probably not important in a normal gravitational environment $f_{0} r$ the conditions corsidered for water and liquid metals.

The theoretical approach, outlined above, to tackle surface-tension driven flows has been applied to study the problem of transient thermocapillary flow in infinitely thin liquid layer with spatially varying temperature distribution imposcd on the free surface (Pimputkar and Ostrach 1980). The layer is assumed thin enough so that, the inertial forces are negligible. The equations of motion arc non-dimensionalized by the scaling procedure described above. Small-time and large-time solutions are obtained with surface height as part of the solution. One potential area of application of such work is the design of experiments and interpretation of data where thermocapillary flows are studied.

\section{Thermo and diffusocapillary forces}

Relatively less study has been made of flows induced by surface tension gradients along the free surface than of those with the gradients normal to it (Marangoni instability). Since the former have many interesting and complex features (like natural convection) and are also of considerable technological importance, increased research on such problems is warranted.

To serve as a guide for future work a review of representative existing work on this type of problem was presented by Ostrach (1977). It is pointed out therein that even for the rather simple thin-layer configuration thcre are inconsistencies in the salutions and uncertainty in the interpretation of the results. Many of the difficultics arise because of the multiplicity of relevant parameters and they could have been avoided if the mathematical models were obtained from the normalization of the complete equations rather than in an ad hoc manner.

In order to differentiate among different types of problems it is important to note that there are two distinet types of diffusacapillary fiows. The first (analogous to thermocapillary flows) occurs because of concentration gradients on the surface and within the bulk of the fluid. The second type (treated in Yih 1969 and Adler and Sowerby 1970) considers an insoluble surface layer. This leads to 
surface-tension gradients but there are no buoyancy effects. This may be significant to plan ground-based experiments. The second type of diffusocapillary phenomena could possibly approach the first type if there is sufficient time for the solute to diffuse into the bulk fluid.

A number of papers (Sirignano and Glassman 1970 ; Adler 1970, 1975; Sharma and Sirignano 1971 ; Torrance and Mahajan 1975), on problems of the general type considered herein have been written in relation to flame spreading phenomena. These are interesting because they deal with problems over different ranges of parametric values, conditions not directly spplicable to space processing. However, their significance lies in the fact that some work is numerical, some analytical, and some experimental and comparisons among them are sometimes made. Thus, indications of physical mechanisms and chccks of assumptions are presented that give further insight into problems of this type.

It appears that relatively little experimental wark has bcen done in these types of problems. Details of on such experiment by the present author, are described in a later article in these proceedings.

\section{Conclasion}

Attention has been facussed on flows driven by surface-tension gradients along the free surface because they are both interesting and important. Some of the complexities of both physics and mathematics have been indicated. A useful method of dyaling with the difficulties was outlined and similarities between flows due to buoyancy and surface-tension gradients was pointed out. The conditions for their relative interaction were derived.

\section{References}

Adler J 1970 Combust. Sci. Technol. 2105

Adler $J$ and Sowerby L $1970 \mathrm{~J}$. Fluid Mech. 42 Pt. 3

Adler J 1975 Int. J. Engg: Sci. 131017

Kenning D B R 1968 Appl. Mech. Rev. 2111

Levich V G 1962 Physicochemical hydrodynamics (Englewood Cliffs, N.J. Prentice Hall)

Lèvich V G and Krylov V S 1969 Adv. Fluid Mech. 1293

Ostrach S 1964 Theory of laminar flows, high speed aero dynamics and jet propulsion (ed.) F K Moore (Princeton University Press) 4528

Ostrach S 1966 Proc. III Int. Heat. Trans. Conf. Vol, 6 AIChE 31

Ostrach S 1977 Physico-chemical hydrodynamics (ed.) V G Levich Festschrist, Vol. II, (Advance Publ. Ltd.) p. 571

Ostrach S 1977 Progr. Astronaut. Aeronaut. (ed.) L Steg 52 AIAA

Pimputkar S M and Ostrach S 1980 Phys. Fluids 231281

Schmidt R J and Milverton S W 1935 Proc. R. Soc. (London) A152

Scriven L E 1974 Drops and bubbles; Proc. Int. Colloq. (eds.) D J Collins, M S Plesset, M M Saffren (Caltech. and Jet Propulsion Lab.) Vol. 1

Sharma O P and Sirignano W A 1971 AIAA 9th Aerospace Science Meeting (New York) Jan. 25-27

Sirignano W A and Glassman I 1970 Cumbust. Sci. Technol. 1307

Stanek V and Szekely J 1964 Chem. Eng. Sci. 25699

Sterning C V and Scriven L E 1959 AlChE J. 5514

Torrance K E and Mahajan R L 1975 Combust. Sci. Technol. 1125

Yih C S 1969 Phys. Fluids 1210 